Mathematical Modelling AND ANALysis

Volume 17 Number 2, April 2012, 161-170

http://dx.doi.org/10.3846/13926292.2012.655788

(c) Vilnius Gediminas Technical University, 2012
Publisher: Taylor\&Francis and VGTU

http://www.tandfonline.com/TMMA

Print ISSN: 1392-6292

Online ISSN: 1648-3510

\title{
On an Elliptic System of $p(x)$-Kirchhoff-Type under Neumann Boundary Condition
}

\section{Zehra Yucedag, Mustafa Avci and Rabil Mashiyev}

Faculty of Science, Dicle University

21280 Diyarbakir, Turkey

E-mail: zehra@dicle.edu.tr; mavci@dicle.edu.tr

E-mail(corresp.): mrabil@dicle.edu.tr; rabilmashiyev@gmail.com

Received December 31, 2010; revised December 29, 2011; published online April 1, 2012

Abstract. In the present paper, by using the direct variational method and the Ekeland variational principle, we study the existence of solutions for an elliptic system of $p(x)$-Kirchhoff-type under Neumann boundary condition and show the existence of a weak solution.

Keywords: variational method, $p(x)$-Kirchhoff-type equation, variable exponent LebesgueSobolev space, nonlocal problems, Ekeland variational principle.

AMS Subject Classification: 35D05; 35J60; 35J70.

\section{Introduction}

We are concerned with the following problem

$$
\left\{\begin{array}{l}
-M_{1}\left(\int_{\Omega} \frac{1}{p(x)}|\nabla u|^{p(x)} d x\right) \operatorname{div}\left(|\nabla u|^{p(x)-2} \nabla u\right)=f(u, v)+h_{1}(x) \text { in } \Omega \\
-M_{2}\left(\int_{\Omega} \frac{1}{p(x)}|\nabla v|^{p(x)} d x\right) \operatorname{div}\left(|\nabla v|^{p(x)-2} \nabla v\right)=g(u, v)+h_{2}(x) \text { in } \Omega \\
\frac{\partial u}{\partial \eta}=\frac{\partial v}{\partial \eta}=0 \text { on } \partial \Omega
\end{array}\right.
$$

where $\Omega \subset \mathbb{R}^{N}$ is a bounded smooth domain, $p \in C(\bar{\Omega})$ with $1<p(x)<N$, $\eta$ is the unit exterior vector on $\partial \Omega$. The system (1.1) is a generalization of a model, so-called Kirchhoff equation, introduced by Kirchhoff [17]. To be more precise, Kirchhoff established a model given by the equation

$$
\rho \frac{\partial^{2} u}{\partial t^{2}}-\left(\frac{P_{0}}{h}+\frac{E}{2 L} \int_{0}^{L}\left|\frac{\partial u}{\partial x}\right|^{2} d x\right) \frac{\partial^{2} u}{\partial x^{2}}=0,
$$

where $\rho, P_{0}, h, E, L$ are constants, which extends the classical D'Alambert's wave equation, by considering the effects of the changes in the length of the 
strings during the vibrations. A distinguish feature of the Kirchhoff equation (1.2) is that the equation contains a nonlocal coefficient $\frac{P_{0}}{h}+\frac{E}{2 L} \int_{0}^{L}\left|\frac{\partial u}{\partial x}\right|^{2} d x$ which depends on the average $\frac{E}{2 L} \int_{0}^{L}\left|\frac{\partial u}{\partial x}\right|^{2} d x$ of the kinetic energy $\frac{1}{2}\left|\frac{\partial u}{\partial x}\right|^{2}$ on $[0, L]$, and hence the equation is no longer a pointwise identity.

On the other hand, the stationary counterpart of (1.2) is given by

$$
\left\{\begin{array}{l}
-\left(a+b \int_{\Omega}|\nabla u|^{2} d x\right) \Delta u=f(x, u) \text { in } \Omega, \\
u=0 \text { on } \partial \Omega,
\end{array}\right.
$$

which has attracted much attention after Lions' paper [19] in which an abstract framework to the problem was given. For some interesting results we refer to $[4,5,11]$. Moreover, nonlocal boundary value problems like (1.1) can be used for modeling several physical and biological systems where $u$ describes a process which depend on the average of itself, such as the population density $[2,6]$.

We want to remark that in studying the Kirchhoff-type equations establishing conditions on $M$ and $f$ is the key argument. In that context the following condition is typical

$$
M(t) \geq m_{0}>0, \quad \text { for all } t \geq 0 .
$$

In [1], the authors show the following Kirchhoff problem has a positive solution

$$
\left\{\begin{array}{l}
-M\left(\int_{\Omega}|\nabla u|^{2} d x\right) \Delta u=f(x, u) \text { in } \Omega, \\
u=0 \text { on } \partial \Omega
\end{array}\right.
$$

under the conditions (1.4) and

$$
\widehat{M}(t)=\int_{0}^{t} M(s) d s \geq M(t) t, \quad \text { for all } t \geq 0 .
$$

However, according to the original meaning of the $M$, in the Kirchhoff equation (1.5), it should be an increasing function. Then,

$$
\widehat{M}(t)<\int_{0}^{t} M(t) d s=M(t) t, \quad \text { for all } t>0
$$

and therefore, condition (1.6) cannot be satisfied. To overcome these difficulties, the authors assume the following:

There exists $m_{1} \geq m_{0}$ and $t_{0}>0$ such that $M(t)=m_{1}$, for all $t \geq t_{0}$.

In [7], the authors show the following $p$-Kirchhoff equation has positive solutions

$$
\left\{\begin{array}{l}
-M\left(\int_{\Omega}|\nabla u|^{p} d x\right)^{p-1} \Delta_{p} u=f(x, u) \text { in } \Omega \\
u=0 \text { on } \partial \Omega
\end{array}\right.
$$

where $M(t)$ satisfies (1.4) for all $t \in \mathbb{R}^{+}$and

$$
\widehat{M}(t) \geq[M(t)]^{p-1} t, \quad \text { for all } t \geq 0 .
$$


However, the condition imposed on $M$ is far away from the physical sense of the original Kirchhoff equation. Hence, the authors use the similar arguments used in [1]. In [8], by using Krasnoselskii's genus it is showed that (1.7) has infinitely many solutions under the conditions $A t^{\alpha} \leq[M(t)]^{p-1} \leq B t^{\alpha}$ and $Q_{1} t^{q-1} \leq f(x, t) \leq Q_{2} t^{q-1}$ for all $t \geq 0$ and for all $x \in \bar{\Omega}$, where $A, B, \alpha$, $Q_{1}, Q_{2}, q$ positive constants, $q \in\left(p, p^{*}\right)$ and $\alpha p>q$. However, under these assumptions the condition (1.4) fails.

The generalization of Kirchhoff equations to $p(x)$-Kirchhoff equation is a quite new topic, so there exists only a few paper [10]. In [10], Dai and Hao study $p(x)$-Kirchhoff equation

$$
\left\{\begin{array}{l}
-M\left(\int_{\Omega} \frac{|\nabla u|^{p(x)}}{p(x)} d x\right) \operatorname{div}\left(|\nabla u|^{p(x)-2} \nabla u\right)=f(x, u) \text { in } \Omega, \\
u=0 \text { on } \partial \Omega,
\end{array}\right.
$$

and show the existence and multiplicity of solutions of (1.9), where $f: \Omega \times \mathbb{R} \rightarrow$ $\mathbb{R}$ satisfies Carathéodory condition, $M(t)$ satisfies (1.4) and

$$
\exists 0<\mu<1 \text { such that } \widehat{M}(t) \geq(1-\mu) M(t) t .
$$

Even under the constant exponent case, condition (1.10) is weaker than condition (1.8). It forces the authors to deal with more potential functions than [7]; for example, they deal with increasing function $M(t)=a+b t$.

In recent years, an increasing attention has been paid to the study of differential equations and variational problems with nonstandard growth conditions. The main interest in studying such problems arises from the presence of the $p(x)$-Laplace operator $\operatorname{div}\left(|\nabla u|^{p(x)-2} \nabla u\right)$. The $p(x)$-Laplace operator is a generalization of $p$-Laplace operator $\operatorname{div}\left(|\nabla u|^{p-2} \nabla u\right)$ obtained in the case when $p(x) \equiv p$ (constant). It is well known that, comparing with the $p$-Laplace operator, the $p(x)$-Laplace operator possesses more complicated nonlinear properties, for example, it is inhomogeneous and usually it does not have the so-called first eigenvalue, since the infimum of its principle eigenvalue is zero. This causes many problems, some classical theories and methods, such as the Lagrange multiplier theorem and the theory of Sobolev spaces, are not applicable.

The nonlinear problems involving the $p(x)$-Laplace operator are extremely attractive because they can be used to model dynamical phenomenons which arise from the study of electrorheological fluids or elastic mechanics. Problems with variable exponent growth conditions also appear in the modelling of stationary thermo-rheological viscous flows of non-Newtonian fluids and in the mathematical description of the processes filtration of an ideal barotropic gas through a porous medium $[3,16,20,21]$.

In the present paper, we consider an elliptic system of $p(x)$-Kirchhoff-type equation (1.1) and when doing this, we don't apply any kind of mostly-used constraints, i.e., growth or asymptotic conditions or Ambrosetti-Rabinowtz condition etc., to nonlinearities $f(x, u)$ and $g(x, u)$. Instead, using the theory of variable exponent Lebesgue-Sobolev spaces and the Ekeland variational principle, we establish the existence of a weak solution. As far as we are concerned, system of $p(x)$-Kirchhoff-type equation has not been investigated yet. 
Nevertheless, in [9] Corrêa and Nascimento investigated an elliptic system of $p$-Kirchhoff-type equation of (1.1). The present paper can be regarded as a generalization of [9]. However, there are some differences; for example, we assume that $\alpha>0$, hence for $\alpha=1$ our condition (M) (see the main results) coincides with the following condition:

$\left(M^{*}\right) M_{1}, M_{2}: \mathbb{R}^{+} \rightarrow \mathbb{R}$ are continuous functions and there is a positive constant $m_{0}$ such that $M_{1}(t), M_{2}(t) \geq m_{0}>0$, for all $t \geq 0$,

which is assumed in [9]. Moreover, when $\alpha \in(0,1)$ the Kirchhoff functions $M_{1}, M_{2}$ may be singular at $t=0$ in $(\mathbf{M})$ while it is not the case for $\left(M^{*}\right)$.

\section{Preliminaries}

Firstly, we state some basic properties of the variable exponent LebesgueSobolev spaces $L^{p(x)}(\Omega)$ and $W^{1, p(x)}(\Omega)$ (for details, see $[14,15,18]$ ).

Set $L_{+}^{\infty}(\Omega)=\left\{p ; p \in L^{\infty}(\Omega)\right.$, essinf $\left.p(x)>1, \forall x \in \Omega\right\}$. For any $p \in$ $L_{+}^{\infty}(\Omega)$, denote

$$
1<p^{-}:=\operatorname{essinf}_{x \in \Omega} p(x) \leq p(x) \leq p^{+}:=\operatorname{essip}_{x \in \Omega} p(x)<\infty .
$$

Let $p \in L_{+}^{\infty}(\Omega)$. We define the variable exponent Lebesgue space by

$$
L^{p(x)}(\Omega)=\left\{u \mid u: \Omega \rightarrow \mathbb{R} \text { is measurable, } \int_{\Omega}|u(x)|^{p(x)} d x<\infty\right\},
$$

then $L^{p(x)}(\Omega)$ endowed with the norm

$$
|u|_{p(x)}=\inf \left\{\lambda>0: \int_{\Omega}|u(x) / \lambda|^{p(x)} d x \leq 1\right\}
$$

becomes a Banach space. The modular of $L^{p(x)}(\Omega)$ is the mapping $\rho(u)$ : $L^{p(x)}(\Omega) \rightarrow \mathbb{R}$ which is defined by

$$
\rho(u)=\int_{\Omega}|u(x)|^{p(x)} d x, \quad \forall u \in L^{p(x)}(\Omega) .
$$

Proposition 1. [14, 18] If $u, u_{n} \in L^{p(x)}(\Omega), n=1,2, \ldots$, then the following statements are equivalent:

(i) $\quad \lim _{n \rightarrow \infty}\left|u_{n}-u\right|_{p(x)}=0 ; \quad$ (ii) $\quad \lim _{n \rightarrow \infty} \rho\left(u_{n}-u\right)=0$;

(iii) $u_{n} \rightarrow u$ in measure in $\Omega$ and $\lim _{n \rightarrow \infty} \rho\left(u_{n}\right)=\rho(u)$.

Proposition 2. [14, 18] If $u, u_{n} \in L^{p(x)}(\Omega), n=1,2, \ldots$, then we have

(i) $|u|_{p(x)}<1(=1 ;>1) \Leftrightarrow \rho(u)<1(=1 ;>1)$;

(ii) $|u|_{p(x)}>1 \Longrightarrow|u|_{p(x)}^{p^{-}} \leq \rho(u) \leq|u|_{p(x)}^{p^{+}}$;

(iii) $|u|_{p(x)}<1 \Longrightarrow|u|_{p(x)}^{p^{+}} \leq \rho(u) \leq|u|_{p(x)}^{p^{-}}$; 


$$
\begin{aligned}
& \text { (iv) } \quad \lim _{n \rightarrow \infty}\left|u_{n}\right|_{p(x)}=0 \Leftrightarrow \lim _{n \rightarrow \infty} \rho\left(u_{n}\right)=0 ; \\
& \text { (v) } \quad \lim _{n \rightarrow \infty}\left|u_{n}\right|_{p(x)} \rightarrow \infty \Leftrightarrow \lim _{n \rightarrow \infty} \rho\left(u_{n}\right) \rightarrow \infty .
\end{aligned}
$$

The variable exponent Sobolev space $W^{1, p(x)}(\Omega)$ is defined by

$$
W^{1, p(x)}(\Omega)=\left\{u \in L^{p(x)}(\Omega):|\nabla u| \in L^{p(x)}(\Omega)\right\},
$$

with the norm

$$
\|u\|_{1, p(x)}=|u|_{p(x)}+|\nabla u|_{p(x)}, \quad \forall u \in W^{1, p(x)}(\Omega) .
$$

Proposition 3. $[14,18]$ If $1<p^{-}$and $p^{+}<\infty$, then the spaces $L^{p(x)}(\Omega)$ and $W^{1, p(x)}(\Omega)$ are separable and reflexive Banach spaces.

Proposition 4. $[14,18]$ Let $\Omega \subset \mathbb{R}^{N}$ be bounded and $p \in C(\bar{\Omega})$. If $q \in C(\bar{\Omega})$ and $1 \leq q(x)<p^{*}(x)=N p(x) /(N-p(x))$ for any $x \in \bar{\Omega}$, then the embedding $W^{1, p(x)}(\Omega) \hookrightarrow L^{q(x)}(\Omega)$ is compact.

Proposition 5. $[14,18]$ If $p \in L^{\infty}(\Omega)$, the conjugate space of $L^{p(x)}(\Omega)$ is $L^{p^{\prime}(x)}(\Omega)$, where $\frac{1}{p^{\prime}(x)}+\frac{1}{p(x)}=1$. For any $u \in L^{p(x)}(\Omega)$ and $v \in L^{p^{\prime}(x)}(\Omega)$, we have

$$
\left|\int_{\Omega} u v d x\right| \leq\left(\frac{1}{p^{-}}+\frac{1}{\left(p^{-}\right)^{\prime}}\right)|u|_{p(x)}|v|_{p^{\prime}(x)} .
$$

\section{The Main Results}

In the sequel, for the simplicity of notation, we write $X:=W^{1, p(x)}(\Omega) \times$ $W^{1, p(x)}(\Omega)$ and $\widehat{\rho}(\nabla u):=\int_{\Omega} \frac{|\nabla u(x)|^{p(x)}}{p(x)} d x$.

Definition 1. We say that $(u, v) \in X$ is a weak solution of (1.1) if

$$
\begin{aligned}
& M_{1}(\widehat{\rho}(\nabla u)) \int_{\Omega}|\nabla u|^{p(x)-2} \nabla u \nabla \varphi d x=\int_{\Omega}\left(f(u, v)+h_{1}(x)\right) \varphi d x, \\
& M_{2}(\widehat{\rho}(\nabla v)) \int_{\Omega}|\nabla v|^{p(x)-2} \nabla v \nabla \psi d x=\int_{\Omega}\left(g(u, v)+h_{2}(x)\right) \psi d x,
\end{aligned}
$$

for any $(\varphi, \psi) \in X$.

We will study problem (1.1) under the following assumptions:

$(\mathbf{M}): M_{1}, M_{2}: \mathbb{R}^{+} \rightarrow \mathbb{R}$ are continuous functions. There exist real numbers $m_{0}>0$ and $\alpha>0$ such that $M_{1}(t), M_{2}(t) \geq m_{0} t^{\alpha-1}$, for all $t>0$. Note that the Kirchhoff functions $M_{1}, M_{2}$ may be singular at $t=0$ for $\alpha \in(0,1)$

$\left(\mathbf{F}_{1}\right)$ : There is a $C^{1}$-function $F: \mathbb{R}^{2} \rightarrow \mathbb{R}$ such that

$$
\frac{\partial F}{\partial u}(u, v)=f(u, v) \text { and } \frac{\partial F}{\partial v}(u, v)=g(u, v),
$$

for all $(u, v) \in \mathbb{R}^{2}$; 
$\left(\mathbf{F}_{2}\right)$ : There is $k>0$ such that $F(u, v)=F(u+k, v+k)$, for all $(u, v) \in \mathbb{R}^{2}$;

$(\mathbf{h}): h_{1}, h_{2} \in L^{q(x)}(\Omega), \frac{1}{p(x)}+\frac{1}{q(x)}=1,1<q(x)<p^{*}(x)$ such that

$$
\int_{\Omega} h_{1}(x) d x=\int_{\Omega} h_{2}(x) d x=0 .
$$

The energy functional corresponding to problem (1.1) is defined as $I: X \rightarrow \mathbb{R}$,

$$
\begin{aligned}
I(u, v)= & \widehat{M}_{1}(\widehat{\rho}(\nabla u))+\widehat{M}_{2}(\widehat{\rho}(\nabla v)) \\
& -\int_{\Omega} F(u, v) d x-\int_{\Omega} h_{1}(x) u d x-\int_{\Omega} h_{2}(x) v d x
\end{aligned}
$$

for all $(u, v) \in X$, where $\widehat{M}_{i}(t)=\int_{0}^{t} M_{i}(s) d s, i=1,2$. In a standard way, it can be proved that $I \in C^{1}(X \backslash\{(0,0)\}, \mathbb{R})$. Moreover, for every $(u, v) \in$ $X \backslash\{(0,0)\}$, the derivative of $I$ is given by

$$
\begin{aligned}
& \left\langle I^{\prime}(u, v),(\phi, \omega)\right\rangle=M_{1}(\widehat{\rho}(\nabla u)) \int_{\Omega}|\nabla u|^{p(x)-2} \nabla u \nabla \phi d x+M_{2}(\widehat{\rho}(\nabla v)) \\
& \quad \times \int_{\Omega}|\nabla v|^{p(x)-2} \nabla v \nabla \omega d x-\int_{\Omega}\left(f(u, v)+h_{1}(x)\right) \phi d x-\int_{\Omega}\left(g(u, v)+h_{2}(x)\right) \omega d x
\end{aligned}
$$

for any $(\phi, \omega) \in X$. Thus $(u, v) \in X \backslash\{(0,0)\}$ is a weak solution of (1.1) if and only if $(u, v)$ is a nontrivial critical point of $I$.

Our main result is the following theorem:

Theorem 1. Suppose $(\mathbf{M}),\left(\mathbf{F}_{1}\right),\left(\mathbf{F}_{2}\right)$ and $(\mathbf{h})$ hold. Then, problem (1.1) has a weak solution $\left(u_{0}, v_{0}\right) \in X$ if $I$ is differentiable at $\left(u_{0}, v_{0}\right)$.

By the same idea developed by Fan in [13], we can split the space $W^{1, p(x)}(\Omega)$ in the following way. Define

$$
W_{0}=\left\{w \in W^{1, p(x)}(\Omega): \int_{\Omega} w d x=0\right\} .
$$

For $w \in W^{1, p(x)}(\Omega)$, denote $\bar{w}=\frac{1}{|\Omega|} \int_{\Omega} w d x$ and $\widetilde{w}=w-\bar{w}$. Then $w=\widetilde{w}+\bar{w}$, where $\bar{w} \in \mathbb{R}$ and $\widetilde{w} \in W_{0}$. So, $W^{1, p(x)}(\Omega)=W_{0} \oplus \mathbb{R}$. $W_{0}$ is a closed linear subspace of $W^{1, p(x)}(\Omega)$ with codimension 1 .

Proposition 6 [Poincaré inequality]. [13] Let $\Omega \subset \mathbb{R}^{N}$ be bounded and smooth.There exists a positive constant c such that

$$
|w|_{p(x)} \leq c|\nabla w|_{p(x)}, \quad \forall w \in W_{0} .
$$

Proof. We will give a similar proof to Fan's stated in [13]. Arguing by contradiction, assume that there exists a sequence $\left\{w_{n}\right\} \subset W_{0}$ such that $\left|w_{n}\right|_{p(x)} \geq n\left|\nabla w_{n}\right|_{p(x)}$. Without loss of generality we may assume $\left|w_{n}\right|_{p(x)}=1$, then $\left|\nabla w_{n}\right|_{p(x)} \leq \frac{1}{n}$. We may assume, taking a subsequence if necessary, that 
$w_{n} \rightarrow w_{0}$ in $W^{1, p(x)}(\Omega), w_{n} \rightarrow w_{0}$ in $L^{p(x)}(\Omega)$ and $w_{n}(x) \rightarrow w_{0}(x)$ for a.e. $x \in \Omega$. So we have that $\left|w_{0}\right|_{p(x)}=1, \int_{\Omega} w_{0} d x=\lim _{n \rightarrow \infty} \int_{\Omega} w_{n} d x=0$, and $\int_{\Omega}\left|\nabla w_{0}\right|^{p(x)} d x \leq \lim _{n \rightarrow \infty} \int_{\Omega}\left|\nabla w_{n}\right|^{p(x)} d x=0$. It follows that $w_{0} \in W_{0}$ and $\nabla w_{0}=0$, consequently $w_{0}=0$, which contradicts $\left|w_{0}\right|_{p(x)}=1$.

To get the proof of Theorem 1, we will use the following version of the Ekeland variational principle.

Proposition 7. [12] Let $E$ be a Banach space and let $\Psi: E \rightarrow \mathbb{R}$ be a $C^{1}$ function which is bounded from below. Then, for any $\epsilon>0$, there exists $w_{\epsilon} \in E$ such that

$$
\Psi\left(w_{\epsilon}\right) \leq \inf _{E} \Psi+\epsilon \quad \text { and } \quad\left\|\Psi^{\prime}\left(w_{\epsilon}\right)\right\|_{E^{*}} \leq \epsilon .
$$

Lemma 1. The functional $I$ is bounded from below.

Proof. At the beginning, we will show that the energy functional $I$ is well defined. To obtain this, it is enough to verify that the functional $J: X \rightarrow \mathbb{R}$, $J(u, v)=\int_{\Omega} F(u, v) d x$, is well defined. Since $F$ is continuous on $[0, k] \times[0, k]$ and $F(u, v)=F(u+k, v+k)$ for all $(u, v) \in \mathbb{R}^{2}$, we obtain $|F(u, v)| \leq c_{1}$ for all $(u, v) \in \mathbb{R}^{2}$. Hence,

$$
|J(u, v)| \leq \int_{\Omega}|F(u, v)| d x \leq c_{1}|\Omega|, \quad \text { for all }(u, v) \in X,
$$

i.e., $J$ is well defined, where $|\Omega|$ is the Lebesgue measure of $\Omega$. Now, we will show that $I$ is bounded from below. Let $(u, v) \in X$. Then $u$ and $v$ can be written as $u=\widetilde{u}+\bar{u}$ and $v=\widetilde{v}+\bar{v}$, where $\bar{u}, \bar{v} \in \mathbb{R}$ and $\widetilde{u}, \widetilde{v} \in W_{0}$ with $\int_{\Omega} \widetilde{u} d x=\int_{\Omega} \widetilde{v} d x=0$. Therefore,

$$
\begin{aligned}
& I(u, v)=\widehat{M}_{1}(\widehat{\rho}(\nabla u))+\widehat{M}_{2}(\widehat{\rho}(\nabla v))-\int_{\Omega} F(\widetilde{u}+\bar{u}, \widetilde{v}+\bar{v}) d x \\
& \quad-\int_{\Omega} h_{1}(x)(\widetilde{u}+\bar{u}) d x-\int_{\Omega} h_{2}(x)(\widetilde{v}+\bar{v}) d x \geq \widehat{M}_{1}(\widehat{\rho}(\nabla u))+\widehat{M}_{2}(\widehat{\rho}(\nabla v)) \\
& \quad-c_{1}|\Omega|-\int_{\Omega} h_{1}(x) \widetilde{u} d x-\bar{u} \int_{\Omega} h_{1}(x) d x-\int_{\Omega} h_{2}(x) \widetilde{v} d x-\bar{v} \int_{\Omega} h_{2}(x) d x .
\end{aligned}
$$

If we consider that $h_{1}, h_{2} \in L^{q(x)}(\Omega) ; \widetilde{u}, \widetilde{v} \in L^{p(x)}(\Omega)$ and use Proposition 5 , it follows

$$
I(u, v) \geq \widehat{M}_{1}(\widehat{\rho}(\nabla u))+\widehat{M}_{2}(\widehat{\rho}(\nabla v))-c_{1}|\Omega|-\left|h_{1}\right|_{q(x)}|\widetilde{u}|_{p(x)}-\left|h_{2}\right|_{q(x)}|\widetilde{v}|_{p(x)} .
$$

From the assumption (M) and Proposition 6, we have

$$
\begin{aligned}
I(u, v) \geq & m_{0} \int_{0}^{\frac{1}{p^{+}} \rho(\nabla u)} s^{\alpha-1} d s+m_{0} \int_{0}^{\frac{1}{p^{+}} \rho(\nabla v)} s^{\alpha-1} d s \\
& -c_{3}|\nabla \widetilde{u}|_{p(x)}-c_{4}|\nabla \widetilde{v}|_{p(x)}-c_{2} \\
= & \frac{m_{0}}{\alpha\left(p^{+}\right)^{\alpha}}\left[(\rho(\nabla u))^{\alpha}+(\rho(\nabla v))^{\alpha}\right]-c_{3}|\nabla \widetilde{u}|_{p(x)}-c_{4}|\nabla \widetilde{v}|_{p(x)}-c_{2} .
\end{aligned}
$$


By Proposition 2, we have

$$
\begin{aligned}
I(u, v) \geq & \frac{m_{0}}{\alpha\left(p^{+}\right)^{\alpha}}\left[(\rho(\nabla u))^{\alpha}+(\rho(\nabla v))^{\alpha}\right]-c_{3} \max \left\{(\rho(\nabla \widetilde{u}))^{\frac{1}{p^{-}}},(\rho(\nabla \widetilde{u}))^{\frac{1}{p^{+}}}\right\} \\
& -c_{4} \max \left\{(\rho(\nabla \widetilde{v}))^{\frac{1}{p^{-}}},(\rho(\nabla \widetilde{v}))^{\frac{1}{p^{+}}}\right\} .
\end{aligned}
$$

So, $I$ is bounded from below.

Remark 1. By Lemma 1, it is easy to see that the main difference between the Dirichlet and Neumann problem is related to noncoerciveness of the energy functional corresponding to Neumann problem. So, in that context, Poincaré inequality plays a key role.

Proof of Theorem 1. Since $I \in C^{1}(X, \mathbb{R})$ is weakly lower semi-continuous and bounded from below, from Ekeland variational principle we have $\left(u_{n}, v_{n}\right) \subset X$ such that

$$
I\left(u_{n}, v_{n}\right) \rightarrow \inf _{X} I \quad \text { and } \quad I^{\prime}\left(u_{n}, v_{n}\right) \rightarrow 0 .
$$

By decomposition of $W^{1, p(x)}(\Omega)$, for each $n \in \mathbb{N}$, we can write any $\left(u_{n}, v_{n}\right) \in X$ as $u_{n}=\widetilde{u}_{n}+\bar{u}_{n}$ and $v_{n}=\widetilde{v}_{n}+\bar{v}_{n}$, where $\bar{u}_{n}, \bar{v}_{n} \in \mathbb{R}$ and $\widetilde{u}_{n}, \widetilde{v}_{n} \in W_{0}$ with $\int_{\Omega} \widetilde{u}_{n} d x=\int_{\Omega} \widetilde{v}_{n} d x=0$. Moreover, from (3.2) we have $\left|I\left(u_{n}, v_{n}\right)\right| \leq c_{5}$. Thus, if we use (3.1), it follows that

$$
c_{6} \leq I\left(u_{n}, v_{n}\right) \leq c_{5} .
$$

This means the sequences $\left\{\rho\left(\nabla \widetilde{u}_{n}\right)\right\}$ and $\left\{\rho\left(\nabla \widetilde{v}_{n}\right)\right\}$ are bounded. Using Proposition 2 and Proposition 6 , we obtain that $\left\{\rho\left(\widetilde{u}_{n}\right)\right\}$ and $\left\{\rho\left(\widetilde{v}_{n}\right)\right\}$ are also bounded. Thus, $\left\{\widetilde{u}_{n}\right\}$ and $\left\{\widetilde{v}_{n}\right\}$ are bounded sequences in $W^{1, p(x)}(\Omega)$. In addition, if we choose $\bar{u}_{n}, \bar{v}_{n}$ in the interval $[0, k], u_{n}=\widetilde{u}_{n}+\bar{u}_{n}$ and $v_{n}=\widetilde{v}_{n}+\bar{v}_{n}$, for each $n \in \mathbb{N}$, we can see that $\left\{\widetilde{u}_{n}\right\}$ and $\left\{\widetilde{v}_{n}\right\}$ are bounded sequences in $W^{1, p(x)}(\Omega)$. So, for convenient subsequences, we have $u_{n} \rightarrow u_{0}$ and $v_{n} \rightarrow v_{0}$ in $W^{1, p(x)}(\Omega)$, and therefore

$$
\int_{\Omega} h_{1} u_{n} d x \rightarrow \int_{\Omega} h_{1} u_{0} d x \text { and } \int_{\Omega} h_{2} v_{n} d x \rightarrow \int_{\Omega} h_{2} v_{0} d x .
$$

Using compact embedding, i.e., Proposition 4, we have

$$
u_{n} \rightarrow u_{0} \quad \text { and } \quad v_{n} \rightarrow v_{0} \quad \text { in } L^{q(x)}(\Omega),
$$

and up to subsequences,

$$
u_{n}(x) \rightarrow u_{0}(x) \quad \text { and } \quad v_{n}(x) \rightarrow v_{0}(x) \quad \text { a.e. } x \in \Omega .
$$

Moreover, by continuity of $F$, it follows

$$
F\left(u_{n}(x), v_{n}(x)\right) \rightarrow F\left(u_{0}(x), v_{0}(x)\right) \quad \text { a.e. } x \in \Omega .
$$

Since $\left|F\left(u_{n}(x), v_{n}(x)\right)\right| \leq c$ for all $n \in \mathbb{N}$ and a.e. $x \in \Omega$, by help of the Lebesgue dominated convergence theorem, we obtain

$$
\int_{\Omega} F\left(u_{n}, v_{n}\right) d x \rightarrow \int_{\Omega} F\left(u_{0}, v_{0}\right) d x
$$


From (3.2) we know that

$$
\begin{aligned}
\inf _{X} I= & \lim I\left(u_{n}, v_{n}\right)=\lim \left[\widehat{M}_{1}\left(\widehat{\rho}\left(\nabla u_{n}\right)\right)+\widehat{M}_{2}\left(\widehat{\rho}\left(\nabla v_{n}\right)\right)\right. \\
& \left.-\int_{\Omega} F\left(u_{n}, v_{n}\right) d x-\int_{\Omega} h_{1}(x) u_{n} d x-\int_{\Omega} h_{2}(x) v_{n} d x\right] .
\end{aligned}
$$

Finally, we have to show that $\left\{\widehat{M}_{1}\left(\widehat{\rho}\left(\nabla u_{n}\right)\right)\right\}$ and $\left\{\widehat{M}_{2}\left(\widehat{\rho}\left(\nabla v_{n}\right)\right)\right\}$ are convergent. Considering that $u_{n} \rightarrow u_{0}$ and $v_{n} \rightarrow v_{0}$ in $W^{1, p(x)}(\Omega)$ and using Fatou's Lemma, we get

$$
\widetilde{\rho}\left(\nabla u_{0}\right) \leq \liminf \widetilde{\rho}\left(\nabla u_{n}\right) \quad \text { and } \quad \widetilde{\rho}\left(\nabla v_{0}\right) \leq \liminf \widetilde{\rho}\left(\nabla v_{n}\right) .
$$

Since $\widehat{M}_{1}$ and $\widehat{M}_{2}$ are continuous and increasing, we obtain

$$
\widehat{M}_{1}\left(\widehat{\rho}\left(\nabla u_{0}\right)\right) \leq \lim \widehat{M}_{1}\left(\widehat{\rho}\left(\nabla u_{n}\right)\right),
$$

and similarly

$$
\widehat{M}_{2}\left(\widehat{\rho}\left(\nabla v_{0}\right)\right) \leq \lim \widehat{M}_{2}\left(\widehat{\rho}\left(\nabla v_{n}\right)\right)
$$

Eventually,

$$
\begin{aligned}
\inf _{X} I \geq & \widehat{M}_{1}\left(\widehat{\rho}\left(\nabla u_{0}\right)\right)+\widehat{M}_{2}\left(\widehat{\rho}\left(\nabla v_{0}\right)\right) \\
& -\int_{\Omega} F\left(u_{0}, v_{0}\right) d x-\int_{\Omega} h_{1}(x) u_{0} d x-\int_{\Omega} h_{2}(x) v_{0} d x=I\left(u_{0}, v_{0}\right),
\end{aligned}
$$

which means $I\left(u_{0}, v_{0}\right)=\inf _{X} I$. Hence, $\left(u_{0}, v_{0}\right) \in X$ is a weak solution of problem (1.1) if $I$ is differentiable at $\left(u_{0}, v_{0}\right)$. The proof is completed.

\section{Acknowledgement}

The authors would like to thank the anonymous referees for their helpful suggestions and corrections, which improved the presentation of this paper.

\section{References}

[1] C.O. Alves, F.J.S.A. Corrêa and T.F. Ma. Positive solutions for a quasilinear elliptic equation of Kirchhoff type. Comput. Math. Appl., 49:85-93, 2005. http://dx.doi.org/10.1016/j.camwa.2005.01.008.

[2] D. Andrade and T.F. Ma. An operator equation suggested by a class of stationary problems. Comm. Appl. Nonlinear Anal., 4:65-71, 1997.

[3] S.N. Antontsev and J.F. Rodrigues. On stationary thermo-rheological viscous flows. Ann. Univ. Ferrara Sez. VII Sci. Mat., 52(7):19-36, 2006. http://dx.doi.org/10.1007/s11565-006-0002-9.

[4] A. Arosio and S. Pannizi. On the well-posedness of the kirchhoff string. Trans. Amer. Math. Soc., 348:305-330, 1996. http://dx.doi.org/10.1090/S0002-9947-96-01532-2.

[5] M.M. Cavalcante, V.N. Cavalcante and J.A. Soriano. Global existence and uniform decay rates for the Kirchhoff-Carrier equation with nonlinear dissipation. Adv. Differential Equations, 6:701-730, 2001. 
[6] M. Chipot and J.F. Rodrigues. On a elliptic equation of $p$-Kirchhoff type via variational methods. RAIRO Modelisation Math. Anal., 26(3):447-467, 1992.

[7] F.J.S. A. Corrêa and G.M. Figueiredo. On a elliptic equation of $p$-Kirchhoff type via variational methods. Bull. Austral. Math. Soc., 74:263-277, 2006. http://dx.doi.org/10.1017/S000497270003570X.

[8] F.J.S.A. Corrêa and G.M. Figueiredo. On a $p$-Kirchhoff equation via Krasnoselskii's genus. Appl. Math. Lett., 22:819-822, 2009.

[9] F.J.S.A. Corrêa and R. G. Nascimento. On a nonlocal elliptic system of $p$ Kirchhoff-type under Neumann boundary condition. Math. Comput. Modelling, 49:598-604, 2009. http://dx.doi.org/10.1016/j.mcm.2008.03.013.

[10] G. Dai and R. Hao. Existence of solutions for a $p(x)$-Kirchhoff-type equation. $J$. Math. Anal. Appl., 359:275-284, 2009. http://dx.doi.org/10.1016/j.jmaa.2009.05.031.

[11] P. D'Ancona and S. Spagnolo. Global solvability for the degenerate Kirchhoff equation with real analytic data. Ann. Univ. Ferrara Sez. VII Sci. Mat., 108:247-262, 1992.

[12] I. Ekeland. On the variational principle. J. Math. Anal. Appl., 47:324-353, 1974. http://dx.doi.org/10.1016/0022-247X(74)90025-0.

[13] X. Fan. Eigenvalues of the $p(x)$-Laplacian Neumann problems. Nonlinear Anal., 67:2982-2992, 2007. http://dx.doi.org/10.1016/j.na.2006.09.052.

[14] X.L. Fan, J.S. Shen and D. Zhao. Sobolev embedding theorems for spaces $W^{m, p(.)}(\Omega)$. J. Math. Anal. Appl., 262:749-760, 2001. http://dx.doi.org/10.1006/jmaa.2001.7618.

[15] X.L. Fan and D. Zhao. On the spaces $L^{p(x)}(\Omega)$ and $W^{m, p(x)}(\Omega)$. J. Math. Anal. Appl., 263:424-446, 2001. http://dx.doi.org/10.1006/jmaa.2000.7617.

[16] T.C. Halsey. Electrorheological fluids. Science, 258:761-766, 1992. http://dx.doi.org/10.1126/science.258.5083.761.

[17] G. Kirchhoff. Mechanik. Teubner, Leipzig, Germany, 1883.

[18] O. Kovăčik and J. Răkosnik. On spaces $L^{p(x)}$ and $W^{k, p(x)}$. Czechoslovak Math. J., 41:592-618, 1991.

[19] J.L. Lions. On some equations in boundary value problems of mathematical physics. In Contemporary Developments in Continuum Mechanics and Partial Differential Equations (Proc. Internat. Sympos., Inst. Mat. Univ. Fed. Rio de Janeiro, Rio de Janeiro, 1977), volume 30 of North-Holland Math. Stud., pp. 284-346, 1978.

[20] M. Růžička. Modeling and Mathematical Theory, Lecture Notes in Mathematics. Springer-Verlag, Berlin, 2000.

[21] V.V. Zhikov. Averaging of functionals of the calculus of variations and elasticity theory. Math. USSR. Izv., 9:33-66, 1987.

http://dx.doi.org/10.1070/IM1987v029n01ABEH000958. 